\title{
Serpentine locomotion articulated chain: ANA II
}

\author{
A. M. Cardona' ${ }^{1}$ J. I. Barrero², C. Otálora' \\ and C. Parra ${ }^{1}$ \\ ${ }^{1}$ Pontificia Universidad Javeriana, Facultad de Ingeniería, Departamento Electrónica, Carrera, Bogotá, Colombia \\ ${ }^{2} 5$ Arbor cir, Natick, MA, USA
}

\begin{abstract}
When humanity faces challenges in solving problems beyond their technical resources, and has no foundation to solve a problem, engineering must search for an answer developing new concepts and innovative frameworks to excel these limitations and travel beyond our capabilities. This project "Serpentine locomotion articulated chain: ANA II" is a self-contained robot built to evaluate the behavior of the platform being capable of serpentine movements, in a modular chain mechanical design, based on a master/slave architecture.
\end{abstract}

Key words: Articulated chains, snakelike robots, master-slave architecture.

\section{INTRODUCTION}

Most of the terrestrial mobile robotic systems developed until now use wheels or limbs as their locomotion elements. The interesting part of working with articulated chains is the ability to move through narrow paths where wheeled or legged platforms can develop some problems, opening applications on mobile robotics.

Terrorist attacks and earthquakes show the evidence of the technical failure of the tools used to attend these catastrophes. In these situations the human loss and endangerment of lives are inevitable. To save the victims of the tragedy, we have to add some important tools for subsequent collapses or fires. One of the most important applications of articulated chains could be the victim's rescue in this type of situation, where the process can be accelerated and the number of persons at risk can be diminished. The other application of a snake robot is the exploration of pipelines for maintenance or reparation. Pipelines are narrow paths where a wheeled or legged platform would fail while trying to move, places where an articulated chain will succeed.

This project develops an articulated chain robot with the ability to move using serpentine locomotion. It is not expected that the platform could explore, we only intend to build a platform for future investigations. The article

Corresponding Author:

A. M. Cardona

Pontificia Universidad Javeriana

Facultad de Ingeniería, Departamento Electrónica

Carrera 7 No. 40-62, Bogotá, Colombia

Email: aura.cardona@javeriana.edu.co will present the state of art of the project, the description, and the development of ANA II, as well as the results and the conclusions drawn from this work.

\section{STATE OF THE ART}

The work on articulated chains with serpentine locomotion began in the early 1970s. In 1972, Hirose and Morishima (1997) focused their work on platforms that could perform lateral undulation and later they developed a series of wheeled coupled-mobility device to improve the movement of the articulated chain. They termed the devices as Active Cord Mechanisms or ACMs.

Shan et al (1997) also worked on articulated chains. They focused on work mainly in obstacles accommodation: they used the obstacles to help propel the structure and not only to avoid them. They accomplished this by using a concertina mode that required a great deal of space.

In 1995, the Japanese company, NEC, developed an articulated chain called Orochi. This work was designed mainly to search for survivals after earthquakes and explosions making this process safer. The device used an active universal joint derive from a Hooke's joint developed by Ikeda and Takanashi (1997).

Nilsson and Ojala (1997) of the Swedish Institute for Computer Science in Sweden, as part of the PIRAIA project, developed a new serpentine universal link. This gives the robot the ability to perform some non-snakelike locomotion modes that incorporate rolling movements. The platform would have the possibility to "hug" a tree and then roll up the tree.

Paap et al (1997) and their group at GMD-SNAKE (1999) in Germany developed a snakelike device, which develops real-time control. The structure uses tensors 


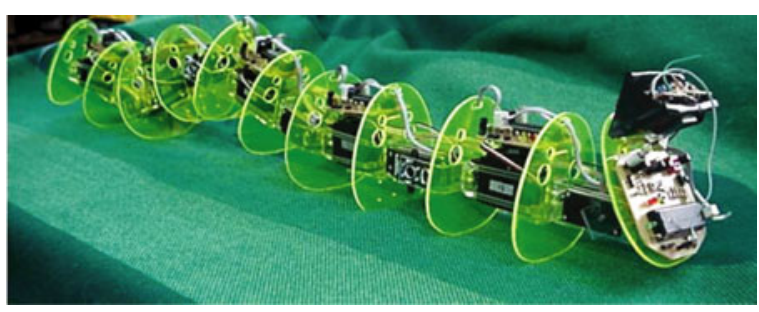

Figure 1 Mechanical structure of ANA II.

combined with a short section of cable winding to give the curvature the device required.

LS Robotics built a snakelike machine called Kaa (Desai et al 1997). It was designed to move through network of pipes and support structures. This was one of the first prototypes of a self-contained unit. It uses RC-servos as actuators; Kaa has a straight-line motion on flat surfaces.

Dr. Gavin Miller of Interval Research Corporation has done some independent work in this area. The work began in 1987 and culminated with the prototype S5 in 1999 with a great resemblance to real snakes (Miller 2000).

These are some of the developments found in the field of snakelike robots. These were the projects that were taken into consideration when developing ANA II for the purpose of this work.

\section{DESCRIPTION}

The final result of the project is the platform of ANA II (see Figure 1). This robot is an articulated chain of five interconnected modular segments. The platform is controlled by a microcontrolled circuit master that has a serial communication (I2C) with the microcontrolled circuits in each of the segments, these are called slaves. These modules are in charge of generating movement sequence and controlling the actuators' positions, which propel each segment of the chain. The master indicates each slave's sequence that must perform to generate the serpentine locomotion.

The serpentine locomotion implemented in ANA II is the rectilinear mode with the capability to make turns and manipulate the direction of the platform (forward or backward).
The development of the project is described in the following section. It is divided in three parts: the description of the mechanical platform, the electronic implementation, and the software of the project.

\section{DEVELOPMENT}

ANA II is a well-built platform that allows one to control the position of each segment in its vertical and horizontal axis. This is possible because of the mechanical structure of each segment built with two actuators (servomotors). The development has given the platform a freedom, which enables it to control and supervise the motion from microcontrolled modules in each segment of the chain. To achieve this goal, the modules were built so that the execution of the motion sequence does not depend on an external computer, but depends on the communication and the information shared between links. A general description of the master/slave modular implementation followed by the sequence description is described below (see Figure 2).

\section{Mechanical platform of ANA II}

The segment structure was made in acrylic (see Figure 3). There is a head segment where the master and the reception module are placed. The segments are numbered from 1 to 5 , one being the head and 5 the tail. The five slave microcontrolled units are interconnected by four wires, the serial communication I2C (SDA, SCL), and the alimentation (5V and ground). ANA II has a total length of $75 \mathrm{~cm}$, a weight of $994 \mathrm{~g}, 10$ degrees of freedom (two per segment), and five segments.

ANA II was conceived and constructed based on the modular concept. It has the advantages of any modular design, easy reproduction, assembly, and maintenance. The platform's modular design allows one to modify the control system of each segment, giving the robot the flexibility to expand its capabilities without any changes in hardware. Also, the platform can be increased or reduced its size without substantial changes in its architecture. Each segment consists of two acrylic "ribs", two servos mounted on acrylic links, a slave or local microcontrolled unit, wiring, screws, and nuts. Each segment has two degrees of freedom, one on the vertical axis and one on horizontal axis.

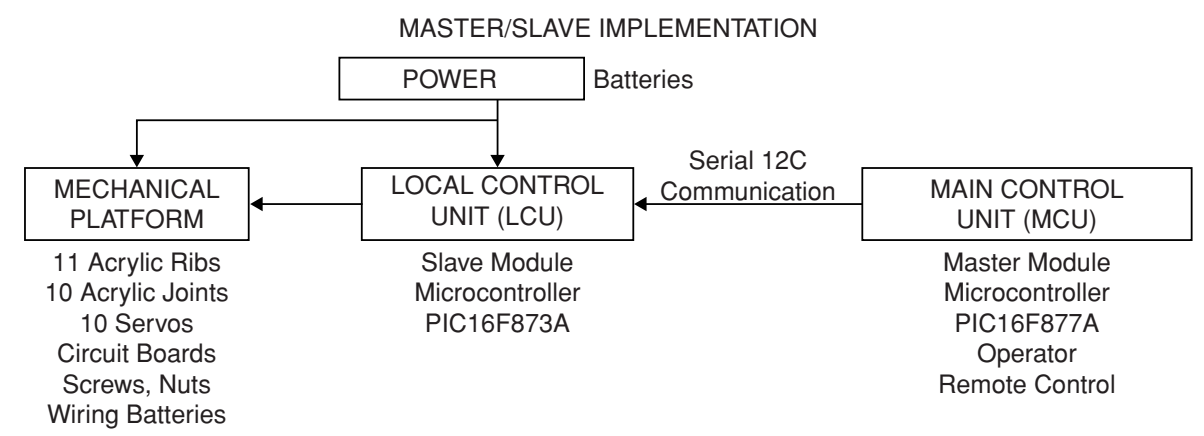

Figure 2 Block diagram of the master/slave implementation. 


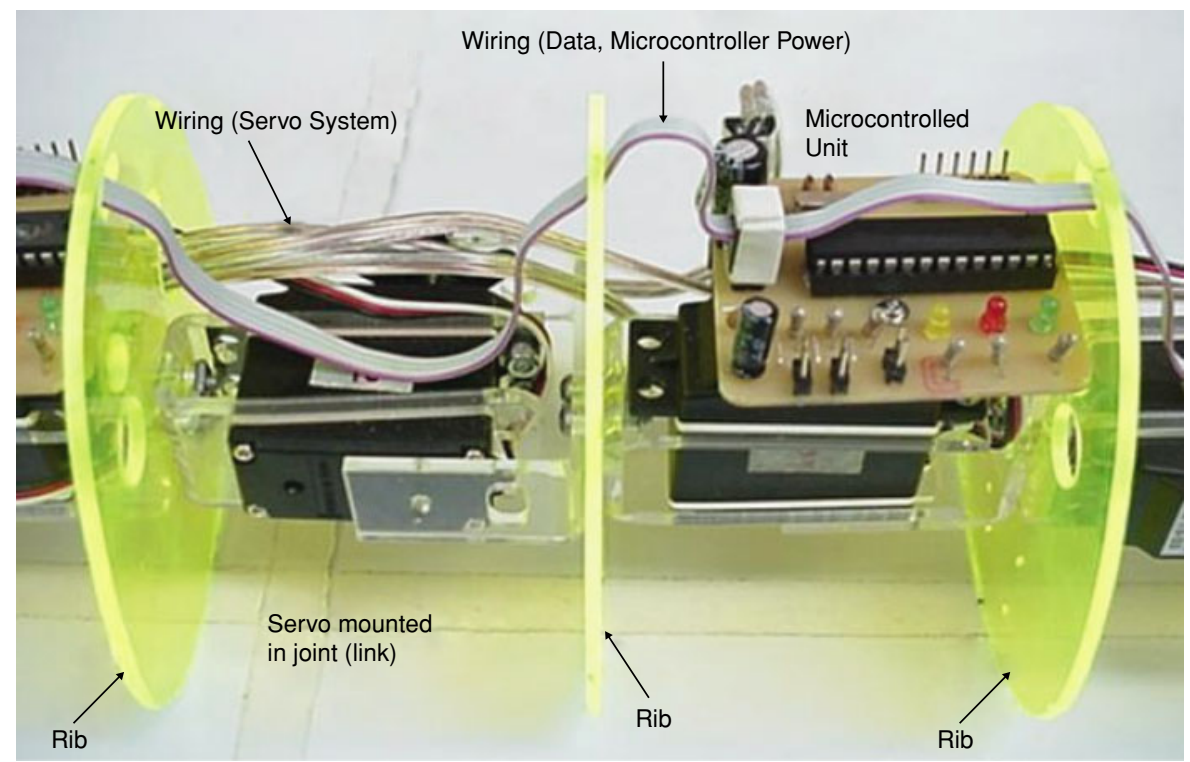

Figure 3 Lateral view of ANA II's segment.

\section{Electronic implementation of ANA II}

The system is constituted by a master unit and five slave units. Each slave unit is in charge of the position control of the segment's servos. The operation of the slave units is independent from each other, and the master unit is in charge of conducting the chain's performance.

The slave units have the capability to handle three contact sensors, and have a free port on the microcontroller for any application. The master unit has the ability to handle a contact sensor, a master reset, different system configuration with a 3-bits dip-switch, a servo control, and a free port on the microcontroller for any application.

Finally, the teleoperation system consists of two parts: the transmission and reception module. The teleoperation module is a commercial one. The reception module installed on the head, receives two impulse signals, which comes from the transmitter. The master unit processes these signals to execute the correspondent sequence.

\section{Control software of motion sequence of ANA II}

Derived from the mathematical studies developed on serpentine locomotion sequence, it can be observed that the movement characteristics that have to be taken into consideration to evaluate the motion of the platform are: magnitude, frequency, and phase (Dowling 1997). Magnitude is the amplitude of the actuator's movement. Frequency is the time an actuator takes to change from one position to another. Phase is the actuator's initial position. These three variables are in constant interaction and affect directly the behavior of the platform.

The master and slave flowcharts are described later.

Figure 4 shows the master module principle flowchart. First, the initial configuration is done. Afterwards, it enters a loop where it waits for the signal coming from the

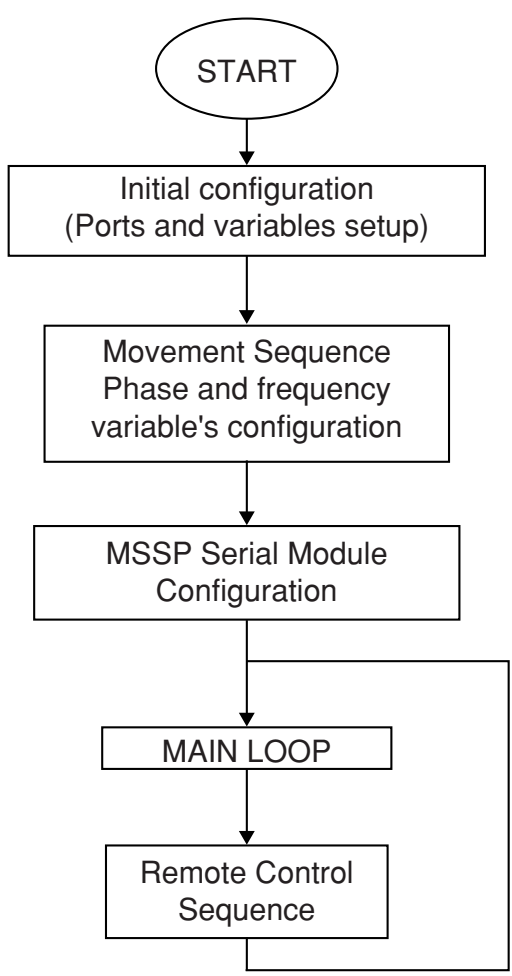

Figure 4 Master module principle flowchart.

transmission module. When the information is received, the master sends the sequence to the slaves.

The subroutine in charge of analyzing the action sent by the operator and the subroutine in charge of sending the information to the slaves is described in the flowcharts of Figure 5.

The serial communication is done by interruption. The master unit indicates when the movement should start, and it does this by interrupting the slave operation so that it 


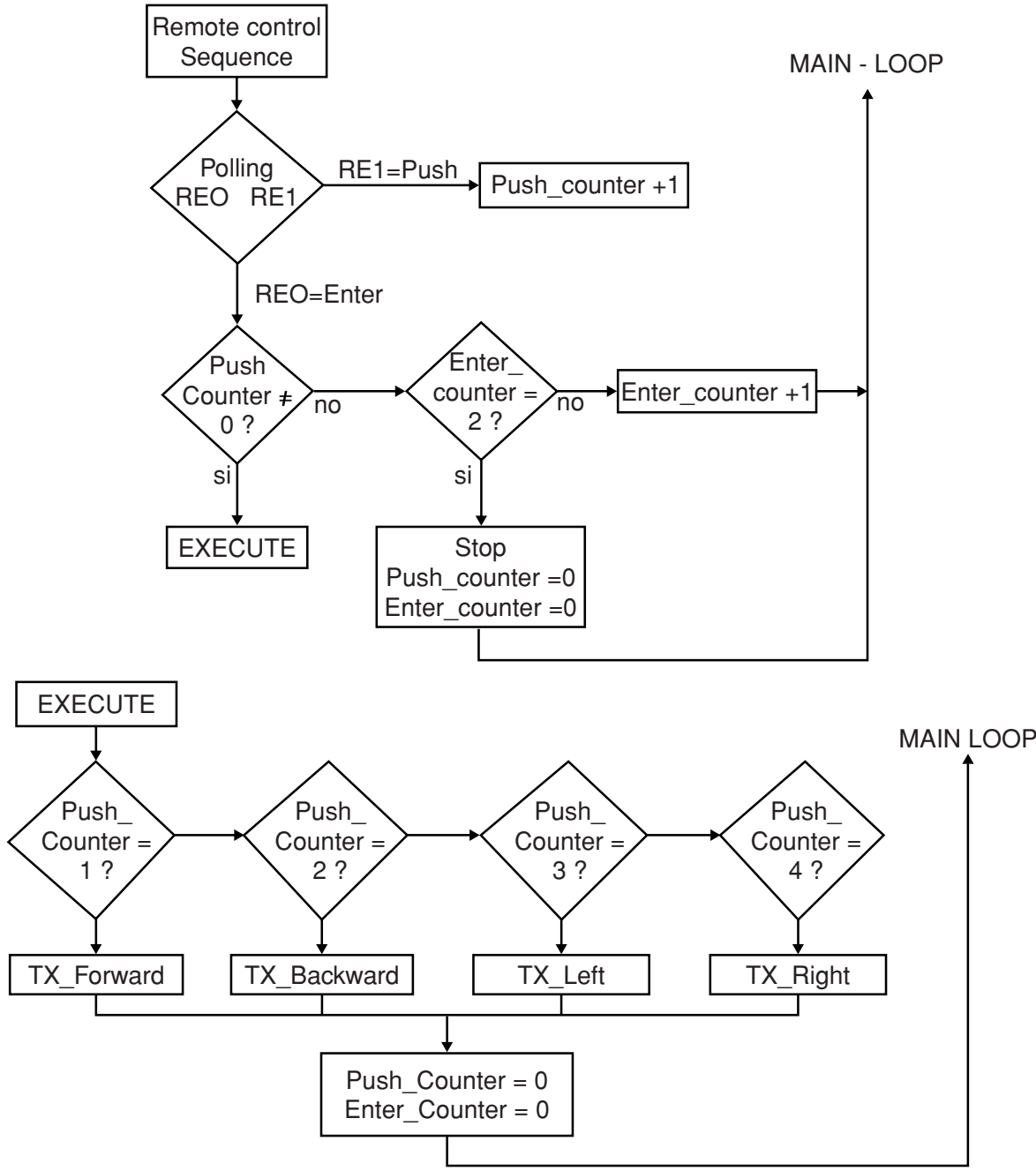

Figure 5 The subroutine in charge of analyzing the action sent by the operator and the subroutine in charge of sending the information to the slaves.

could be attended. The master unit sends 5 byte-streams to each slave unit. Each byte-streams has 5 bytes and each byte has 8 bits. The five bytes sent are:

Direccion (address): it is the address of the slave unit.

Control Estado (state control): it indicates the sequence or command of the operator chosen; forward, backward, left, or right.

Limite Superior (high limit): it indicates the higher limit at which the vertical servo can move. This variable allows one to modify the magnitude of the movement.

Limite Inferior (low limit): it indicates the lower limit at which the vertical servo can move. This variable allows one to modify the magnitude of the movement.

Retardo (delay): it indicates the delay between the position changes of the servo. This variable allows one to modify the frequency of the movement.

Offset: it indicates the initial servo's position. This characterizes the offset of the movement.
Figure 6 shows that the slave unit is always reviewing the command that it is executing at any given time, it allows one to make the necessary changes on each segment when the master unit indicates.

Figure 7 shows the organization of the interruption routine so it can attend three possible interruption sources. The TMR0 in charge of the PWM's duty cycle for each servo. The SSP in charge of the I2C communication with the master unit and the TMR1 in charge of starting the PWM signal period.

\section{Sequence implementation}

After the development of the mechanical structure of ANA II and its microcontrolled system, the serpentine gaits or movement sequences are implemented.

\section{Rectilinear sequence}

The objective of this gait is to simulate the rectilinear gait of serpents (Lissman 1997). The same signal is sent to all the segments, but with a $90^{\circ}$ offset among them. The second segment has a $90^{\circ}$ offset from the first segment, 


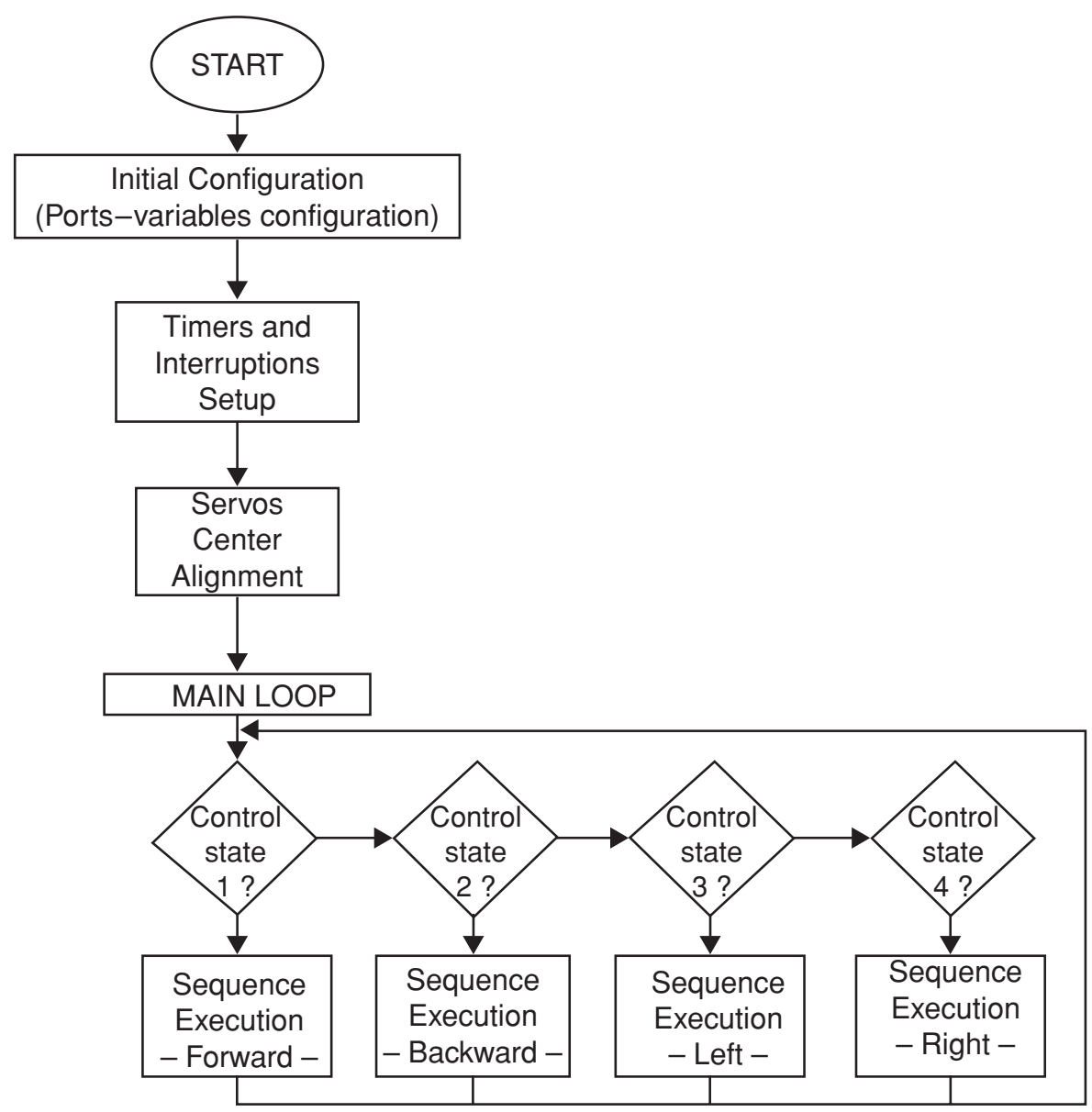

Figure 6 Figure shows that the slave unit is always reviewing the command that it is executing.
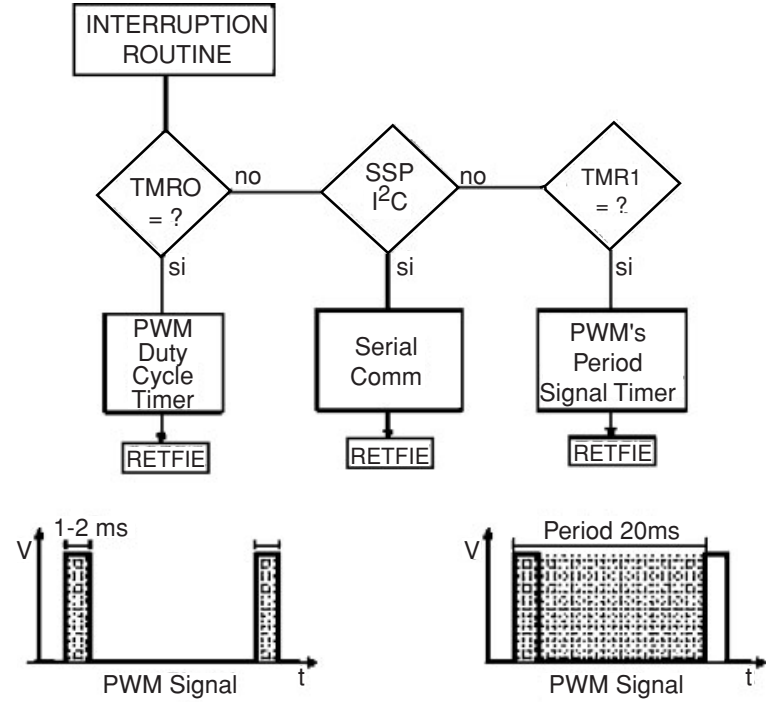

Figure 7 Figure shows the organization of the interruption routine.

the third segment has a $180^{\circ}$ offset from the first segment, the fourth segment has a $270^{\circ}$ offset from the first segment, and the fifth segment has a $360^{\circ}$ offset from the first segment (see Figure 8). These offsets are achieved with setting

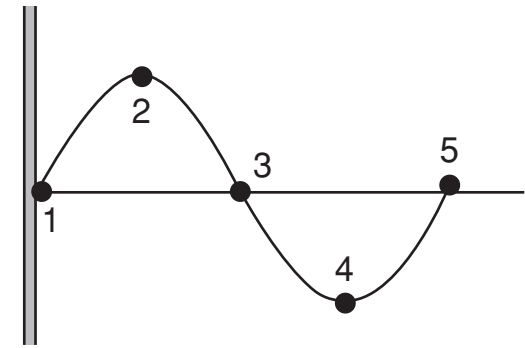

Figure 8 Figure shows the initial points of each segment.

the variable offset or initial position sent to each segment from the master. Thus, a vertical sine wave movement can be generated along the platform. All the segments receive a triangle signal for the vertical servo's movement sequence.

In the rectilinear gait, each segment sends a triangle signal to the vertical servo and a steady center signal to all the horizontal servos. As explained before, the master sends to each servo the initial position. In this sequence the initial position of each segment is: segment no. 1, center; segment no. 2, high limit; segment no. 3 , center; segment no. 4, low limit; and segment no. 5 , center.

Different amplitudes are manipulated varying the vertical servo position from a $0^{\circ}$ to a $180^{\circ}$ range divided 
into 100 steps. This allows a resolution up to $1.8^{\circ}$ per step. These angles are taken from the center located at $90^{\circ}$ toward the edges or mechanical limits of the servo.

\section{Rectilinear sequence with turns}

The objective of this sequence is to evaluate the platform's turning capability. To achieve this, the vertical servos will be receiving the same signal with the same offsets to maintain the platform translation, but now the horizontal servos will move, which characterizes the turning radio, to the right or left of a specific amplitude.

\section{RESULTS}

This type of projects is a challenge because of the complexity of the mechanical arrangement and the involvement of different areas of electronics in robotics (communications, digital systems, electrical circuits, and control) that makes difficult the development of this kind of systems.

The qualitative results of the platform's forward translation are that the speed is directly proportional to the amplitude (more amplitude, faster movement). Also, the power consumption is proportional to the amplitude, therefore proportional to the speed. The faster the platform moves, more energy is needed to supply movement for higher amplitudes. This was an expected result because if the load of the servos increases, also the demand for current will increase. Another result is that as the frequency increases so does the platform speed of translation.

We were able to prove that in order to manipulate the platform's movement direction, the variable needed to be manipulated was the phase in the chain. This was accomplished by changing the direction of the wave moving along the chain, modifying the initial state of the segments, therefore the phase of the wave.

The results obtained by the turning implementation showed that the platform is able to turn in both directions, left and right, with a turning radio of $40 \mathrm{~cm}$. With these tests the current load was increased because of the simultaneous operation of all the servos.

The platform's evaluation and behavior on different surfaces also agreed with the previous results of magnitude and speed. The platform achieved movement in all the surfaces where it was tested. On the smooth, rough, and sand surfaces the platform was able to move forward and backward without any problem. On the stony surface the rectilinear gait was not efficient because the increase of irregularities in the surface decreased the stability of the robot using this particular gait.

One of the advantages of this type of platforms is their capability to keep on working when one of its segments fails, because the other segments will generate enough force to still move the chain, something that rarely happens with wheeled or legged platforms.

The three main variables that allow one to control the serpentine movement in an articulated chain are: magnitude, frequency, and phase. Each of these variables has influence in the final outcome of the sequence and the way the platform moves. The higher the magnitude, the higher the force that each segment adds to the chain to move; thus, the speed increases as the energy needed does too. The higher the frequency, the higher the speed of the movement, but lose to friction with the ground is the limit of this variable. The phase is the variable that characterizes the gait of the platform, indicating the initial position to each segment before the execution of a sequence.

The rectilinear gait is easy to develop because it can be implemented and manipulated by the three variables mentioned in the previous paragraph, in only the vertical axis of the segment. Therefore, it is more complicated to develop an undulatory gait, which is the most common serpentine gait, because it combines movement in the vertical axis and the horizontal axis.

\section{SUMMARY}

The project gives an experimental mechanical foundation (modular structure) and a well-documented robot to develop a complete robotic system capable of performing advanced operations in the articulated chain field. The platform's modular design allows one to modify the control system of each segment, giving the robot the flexibility to expand its capabilities without any changes in hardware. Besides, it has the advantages of any modular design, easy reproduction, assembly, and maintenance. The number of segments is limited by the servo's torque. If the chain is too long, the middle chain servos have to be strong enough to move. This also is related to the platform's weight, a variable that has to be always in mind.

This project opens an endless list of applications in which the human kind is incapable to operate or it is dangerous to perform. Today, the technology is a tool powered by imagination and the human perseverance. Thus, the small step that has been taken is a potential base to research and chain robot's development. In a short-term period the platform can be adapted to implement more efficient locomotion methods and a remote control capable of varying the magnitude and the frequency of the movement. A video camera system can be installed on the robot for visual feedback for exploration purposes. Also, the system can be upgraded with a sensor array to control servo current, obstacles, and different surfaces. The battery system can also be upgraded with the latest technology (light). The servos can be lighter and with low current high torque specifications. Some research in shields or skins to cover the platform can be done, in order to provide the platform with heat resistant, water resistant, or corrosive agents resistant capabilities.

In a long-term the project can reach the stage of a fully functional exploration and recognition system, pipe maintenance, search and rescue system for disasters, military intelligence system, etc. 


\section{REFERENCES}

Desai R, Rosenberg CJ, Jones JL. 1997. Kaa: An autonomous serpentine robot utilizes behavior control. In: Dowling K (Ed.), Limbless Locomotion: Learning to Crawl with a Snake Robot. Thesis (Doctor of Philosophy in Robotics), Carnegie Mellon University, The Robotics Institute, Pittsburgh, p. 31.

Dowling K. 1997. Limbless Locomotion: Learning to Crawl with a Snake Robot. Thesis (Doctor of Philosophy in Robotics), Carnegie Mellon University, The Robotics Institute, Pittsburgh, pp. 61-6.

GMD-SNAKE. 1999. Robot-snake with flexible real-time control. GMD's Institute for Autonomous Intelligent Systems. http://ais.gmd.de/BAR/snake.html

Hirose S, Morishima A. 1997. Design and control of a mobile robot with an articulated body. In: Dowling K (Ed.), Limbless Locomotion: Learning to Crawl with a Snake Robot. Thesis (Doctor of Philosophy in Robotics), Carnegie Mellon University, The Robotics Institute, Pittsburgh, pp. 21-4.

Ikeda H, Takanashi N. 1997. Joint assembly moveable like a human arm US Patent 4683406. In: Dowling K (Ed.), Limbless Locomotion: Learning to Crawl with a Snake Robot. Thesis (Doctor of Philosophy in Robotics), Carnegie Mellon University, The Robotics Institute, Pittsburgh, pp. 27-8.
Lissman HW. 1997. Rectilinear motion in a snake ( $B o a$ occidentalis). In: Dowling K (Ed.), Limbless Locomotion: Learning to Crawl with a Snake Robot. Thesis (Doctor of Philosophy in Robotics), Carnegie Mellon University, The Robotics Institute, Pittsburgh, pp. 17-8.

Miller G. 2000. Snake robots. Private research project into snake locomotion started in 2000. http://www.snakerobots.com/

Nilsson M, Ojala J. 1997. Self-awareness in reinforcement learning of snake like robot locomotion. In: Dowling K (Ed.), Limbless Locomotion: Learning to Crawl with a Snake Robot. Thesis (Doctor of Philosophy in Robotics), Carnegie Mellon University, The Robotics Institute, Pittsburgh, p. 29.

Paap KL, Dehlwisch M, Klaassen B. 1997. GMD-Snake: A semi-autonomous snake like robot. In: Dowling K (Ed.), Limbless Locomotion: Learning to Crawl with a Snake Robot. Thesis (Doctor of Philosophy in Robotics), Carnegie Mellon University, The Robotics Institute, Pittsburgh, p. 30.

Shan Y et al. 1997. Design and motion planning of a mechanical snake. In: Dowling K (Ed.), Limbless Locomotion: Learning to Crawl with a Snake Robot. Thesis (Doctor of Philosophy in Robotics), Carnegie Mellon University, The Robotics Institute, Pittsburgh, pp. 26-7. 

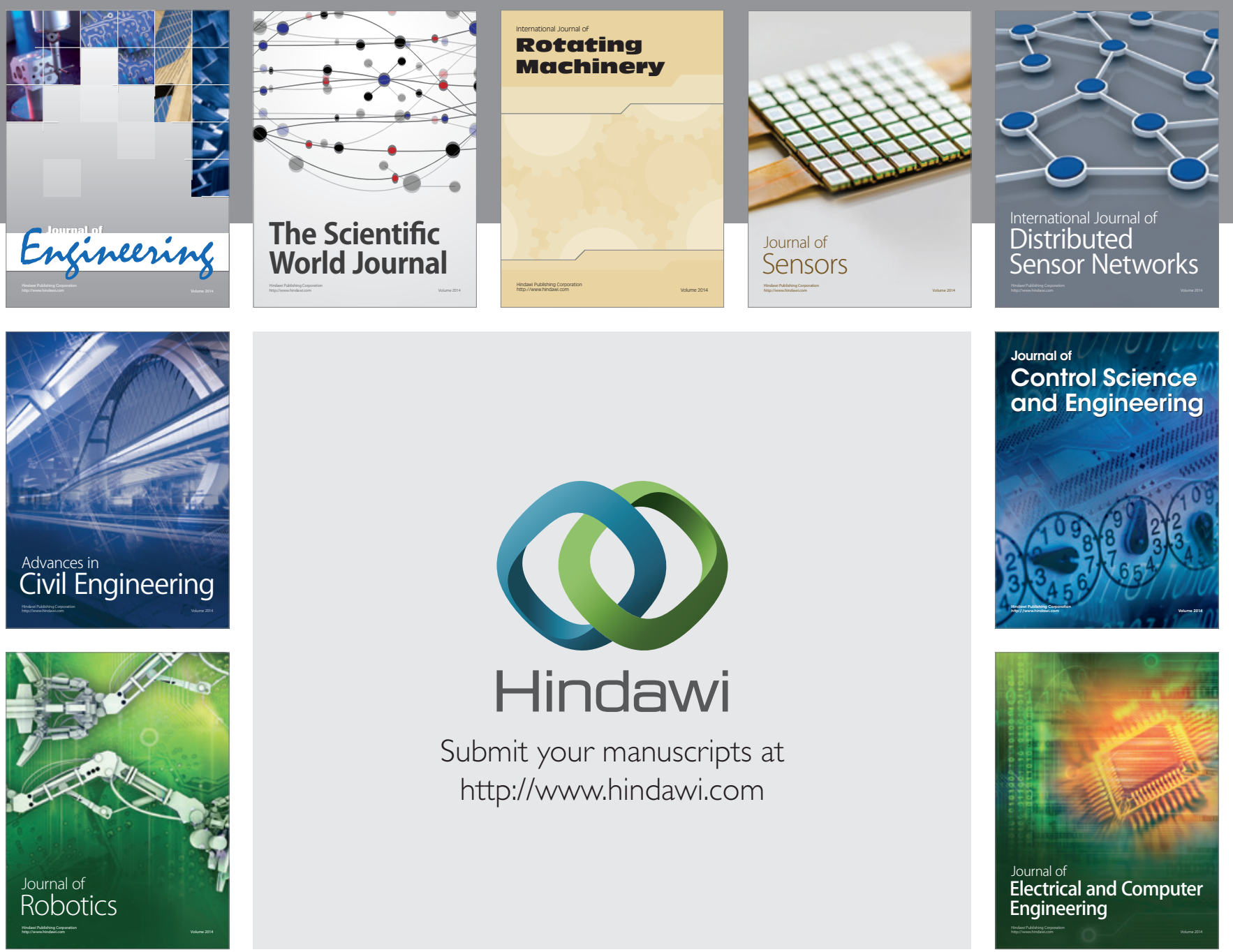

Submit your manuscripts at

http://www.hindawi.com
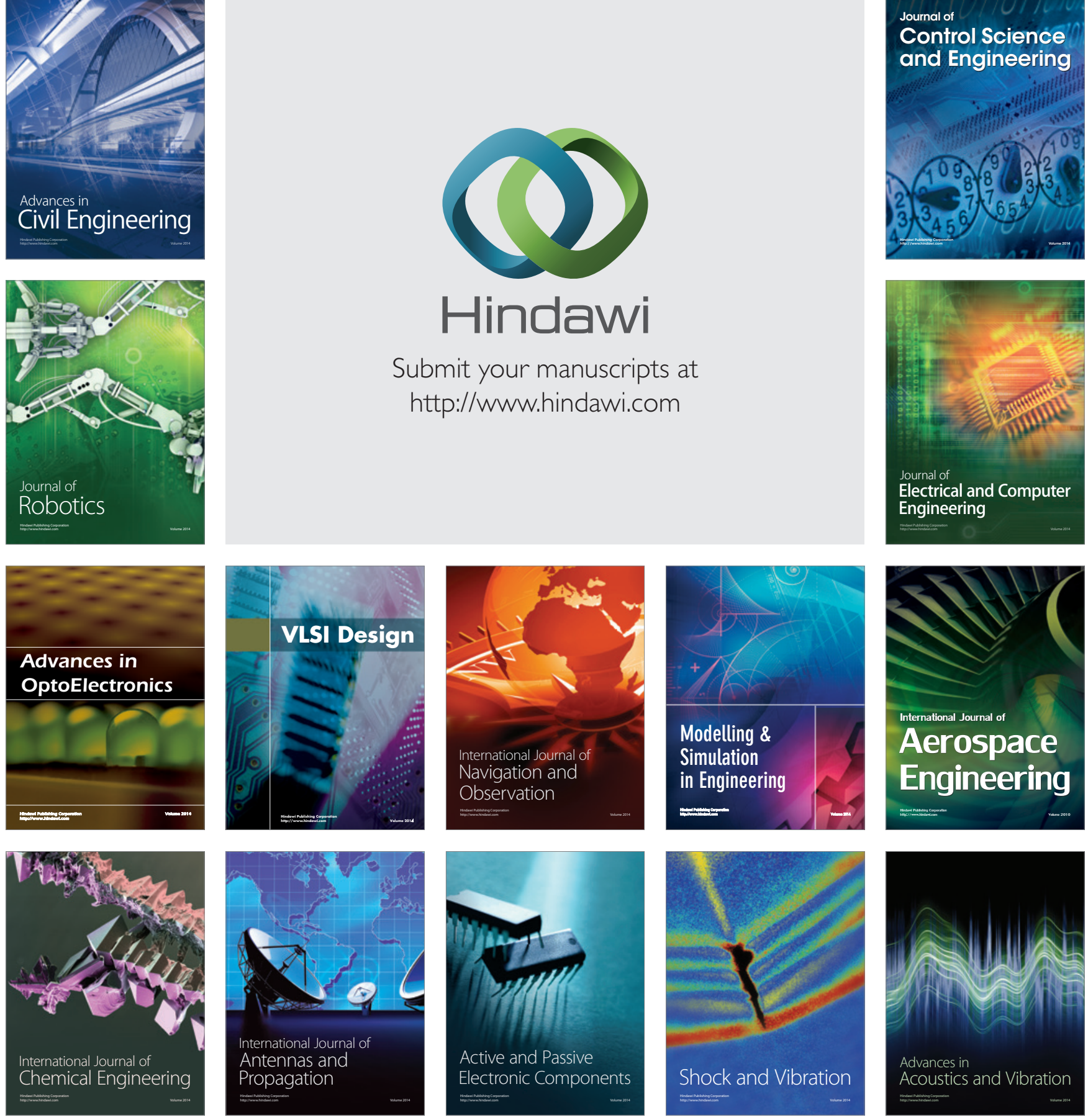